\title{
Fully Relativistic Full-Potential Calculations of Magnetic Moments in Uranium Monochalcogenides with the Dirac Current
}

\author{
Shugo Suzuki and Hidehisa OHTA \\ Institute of Materials Science, University of Tsukuba, Tsukuba 305-8573
}

(Received April 26, 2010)

\begin{abstract}
We study the orbital, spin, and total magnetic moments in uranium monochalcogenides, $\mathrm{U} X$ where $X=\mathrm{S}$, Se, and Te, using the fully relativistic full-potential calculations based on the spin density functional theory. In particular, the orbital magnetic moments are calculated with the Dirac current. We employ two methods which adopt distinctly different basis sets; one is the fully relativistic full-potential linear-combination-of-atomic-orbitals (FFLCAO) method and the other is the fully relativistic full-potential mixed-basis (FFMB) method. Showing that the orbital magnetic moments calculated using the FFLCAO method and those calculated using the FFMB method agree very well with each other, we demonstrate that, in contrast to the conventional method, the method with the Dirac current enables us to calculate the orbital magnetic moments even if the basis set includes basis functions with no definite angular momenta, e.g., the plane waves in the FFMB method. Furthermore, it is found that the orbital magnetic moments obtained in this work are larger by nearly 0.4 $\mu_{\mathrm{B}}$ than those obtained using the conventional method. This is crucial because the resultant differences in the total magnetic moments are about $30 \%$. We compare the results of this work with those of previous theoretical and experimental studies.
\end{abstract}

KEYWORDS: Dirac current, orbital magnetic moment, fully relativistic calculations, fullpotential calculations, uranium monochalcogenide, spin density functional theory

\section{Introduction}

For more than a half century, the properties of actinide compounds have been studied both experimentally and theoretically. ${ }^{1)}$ Among them, uranium monochalcogenides, $\mathrm{U} X$ where $X=$ $\mathrm{S}, \mathrm{Se}$, and Te, have been studied extensively as a typical material. In particular, their magnetic properties have attracted much attention. ${ }^{2-11)}$ The experimental studies have revealed that $\mathrm{UX}$ are ferromagnetic at low temperatures. One remarkable feature of the ferromagnetism in $\mathrm{UX}$ is that it is the orbital magnetic moments, $M^{\text {orb }}$, that dominate in the total magnetic moments, $M^{\text {tot }}$, overcoming the spin magnetic moments, $M^{\text {spin }}{ }^{12)}$

The calculation of $M^{\text {orb }}$ is subtle in contrast to the calculation of $M^{\text {spin }}$; for the latter quantity, we need only to integrate the spin density. So far, for calculating $M^{\text {orb }}$, a conventional method has been widely used. ${ }^{13)}$ In this method, the magnetic-moment operator is defined in an appropriate way, and $M^{\text {orb }}$ are then calculated using the expectation values of the magnetic-moment operator in the Bloch states. In actual calculations, the evaluation of the 
elements of the magnetic-moment operator requires little more than a rearrangement of the overlap matrix. The magnetic moments of the unit cell calculated using this method can be partitioned into the magnetic moments of the constituent atoms or atomic orbitals attributing each basis function to the atom or atomic orbital to which the basis function belongs. A disadvantage of this method is that one cannot use basis functions with no definite angular momenta, e.g., plane waves.

Another method for calculating the magnetic moments is to use the current density as described in the textbooks of electrodynamics. ${ }^{14)}$ That is, if the current density is obtained, one can calculate the magnetic moments by integrating the cross product between the position vector and the current density. For $\mathrm{U} X$ as well as other actinide compounds, since the relativistic effects are significant, the calculation of the current density should be performed using the Dirac current because this includes all the relativistic effects. In the fully relativistic calculations based on the density functional theory adopting a single-particle equation of the Kohn-Sham-Dirac type, the Dirac current is calculated simply using the Dirac matrices. Furthermore, in contrast to the conventional method for calculating $M^{\text {orb }}$, this method has an advantage that the procedure can be applied even if the basis set includes basis functions with no definite angular momenta. This is favorable because the physical quantities should be calculated whatever the basis set is if its quality is good. However, to our knowledge, the calculation of the magnetic moments in $\mathrm{U} X$ as well as other actinide compounds with the Dirac current has not been reported so far. Thus, it seems interesting to compare $M^{\text {orb }}$ calculated using the conventional method and those calculated using the method with the Dirac current.

When applying the method with the Dirac current, the following point should be noted. Since the integral of the cross product between the position vector and the Dirac current does not converge if the integral is performed over an infinitely extended system, as is the case for a crystalline solid, because of the position vector in the integrand. For this reason, an appropriate atomic partitioning scheme is needed. One natural choice is to use the Voronoi cells. In actual calculations, since the Voronoi cells with sharp boundaries are not suitable for accurate numerical calculations, the Voronoi cells with smooth boundaries are useful instead. ${ }^{15-17)}$

It is worth pointing out that, strictly speaking, there is no guarantee of reproducing $M^{\text {orb }}$ if one employs the spin density functional theory (SDFT), the framework used widely so far, in which only the electron density and the spin density are taken as basic variables. The theory that takes the current density as an additional basic variable has been developed, known as the current density functional theory (CDFT). ${ }^{18-23)}$ Although, even within SDFT, $M^{\text {orb }}$ in $\mathrm{UX}$ induced by spin-orbit coupling largely contribute to $M^{\text {tot }}, M^{\text {orb }}$ are most likely enhanced considerably when taking account of the exchange-correlation effects due to the current density as shown for $3 d$ ferromagnetic metals. ${ }^{24)}$ On the other hand, even if we restrict ourselves within SDFT, it seems important to compare $M^{\text {orb }}$ calculated using the two different methods, i.e., 
the conventional method and the method with the Dirac current; since all the calculations of $M^{\text {orb }}$ in UX reported so far have been performed within SDFT, the restriction within SDFT at this stage may be useful for unambiguous comparison and also for step-by-step progress.

In this work, we study $M^{\text {orb }}, M^{\text {spin }}$, and $M^{\text {tot }}$ in UX using the fully relativistic fullpotential calculations. In particular, $M^{\text {orb }}$ are calculated with the integral of the cross product between the position vector and the Dirac current. The calculations are performed with the fully relativistic full-potential linear-combination-of-atomic-orbitals (FFLCAO) method and the fully relativistic full-potential mixed-basis (FFMB) method, both based on SDFT within the local spin density approximation (LSDA).${ }^{25)}$ With the two methods, whose basis sets are distinctly different from each other, we can examine the reliability of the results with respect to the quality of basis sets. In $\S 2$, we describe the method of calculations. The results and discussion are given in $\S 3$. Here, we compare the results of the FFLCAO calculations and those of the FFMB calculations. We also compare the results of this work with those of previous theoretical and experimental studies. Finally, we give the conclusions of this work in $\S 4$.

\section{Method of Calculations}

We begin with the following self-consistent equations:

$$
\begin{gathered}
{\left[c \boldsymbol{\alpha} \cdot \boldsymbol{p}+(\beta-I) m c^{2}+V_{\mathrm{es}}(\boldsymbol{r})+V_{\mathrm{xc}}(\boldsymbol{r})+\beta \boldsymbol{\Sigma} \cdot \boldsymbol{B}_{\mathrm{xc}}(\boldsymbol{r})\right] \psi_{\nu}(\boldsymbol{r})=\varepsilon_{\nu} \psi_{\nu}(\boldsymbol{r}),} \\
\rho(\boldsymbol{r})=-e \sum_{\nu} f_{\nu} \psi_{\nu}(\boldsymbol{r})^{\dagger} \psi_{\nu}(\boldsymbol{r})
\end{gathered}
$$

and

$$
\boldsymbol{m}(\boldsymbol{r})=-e \sum_{\nu} f_{\nu} \psi_{\nu}(\boldsymbol{r})^{\dagger} \beta \boldsymbol{\Sigma} \psi_{\nu}(\boldsymbol{r})
$$

In the Dirac Hamiltonian in the left-hand side of eq. (1), $c$ and $m$ denote the speed of light and the rest mass of an electron, respectively, and the rest energy of an electron, $m c^{2}$, is subtracted. Also, $\boldsymbol{\alpha}$ and $\beta$ are the Dirac matrices in the usual representation. ${ }^{26)}$ In the self-consistent equations, the four-component spinor $\psi_{\nu}(\boldsymbol{r})$ is the one-electron wave function of the $\nu$ th level with the energy eigenvalue $\varepsilon_{\nu}$ and the occupation number $f_{\nu}$; for a crystalline solid, $\nu$ represents the band index $n$ and the wave vector $\boldsymbol{k}$. In eq. (1), $V_{\mathrm{es}}(\boldsymbol{r})$ is the electrostatic potential originated in the nuclear charges and the electron charge density, where the latter is denoted by $\rho(\boldsymbol{r})$ in eq. (2) with $e$ being the electron charge. Also, $V_{\mathrm{xc}}(\boldsymbol{r})=\left[V_{\mathrm{xc}}^{\mathrm{up}}(\boldsymbol{r})+V_{\mathrm{xc}}^{\text {down }}(\boldsymbol{r})\right] / 2$ is the effective scalar potential that describes the spin-independent part of the exchange-correlation potential and $\boldsymbol{B}_{\mathrm{xc}}(\boldsymbol{r})=\left[V_{\mathrm{xc}}^{\text {up }}(\boldsymbol{r})-V_{\mathrm{xc}}^{\text {down }}(\boldsymbol{r})\right] / 2 \boldsymbol{e}_{z}$ is the effective magnetic field that describes the spin-dependent part of the exchange-correlation potential, where $V_{\mathrm{xc}}^{\mathrm{up}}(\boldsymbol{r})$ and $V_{\mathrm{xc}}^{\text {down }}(\boldsymbol{r})$ represent the exchange-correlation potentials for up- and down-spin electrons, respectively, and $\boldsymbol{e}_{z}$ represents the unit vector along the $z$ axis; $\boldsymbol{B}_{\mathrm{xc}}(\boldsymbol{r})$ is originated in the spin magnetization density, $\boldsymbol{m}(\boldsymbol{r})$, which is calculated with $\boldsymbol{\Sigma}=I_{2} \otimes \boldsymbol{\sigma}$ where $I_{2}$ is the $2 \times 2$ unit matrix and 
$\boldsymbol{\sigma}$ are the usual $2 \times 2$ Pauli spin matrices. The electron charge density $\rho(\boldsymbol{r})$ and the spin magnetization density $\boldsymbol{m}(\boldsymbol{r})$ are calculated with $\psi_{\nu}(\boldsymbol{r})$ and $f_{\nu}$. The Dirac current is then obtained with the following equation:

$$
\boldsymbol{j}(\boldsymbol{r})=-e \sum_{\nu} f_{\nu} \psi_{\nu}(\boldsymbol{r})^{\dagger} c \boldsymbol{\alpha} \psi_{\nu}(\boldsymbol{r})
$$

It is crucial to note that $\boldsymbol{j}(\boldsymbol{r})$ consists of not only the orbital contribution but also the spin contribution according to the Gordon decomposition. ${ }^{27)}$

To divide $\boldsymbol{m}(\boldsymbol{r})$ and $\boldsymbol{j}(\boldsymbol{r})$ into atomic components, we use the atomic partitioning scheme adopting the Voronoi cells with smooth boundaries. ${ }^{15-17)}$ In this scheme, the weight function associated with the ath atom, $w_{a}(\boldsymbol{r})$, is introduced as follows:

$$
\sum_{a} w_{a}(\boldsymbol{r})=1
$$

where

$$
w_{a}(\boldsymbol{r})=p\left(\boldsymbol{r}-\boldsymbol{r}_{a}\right) / \sum_{b} p\left(\boldsymbol{r}-\boldsymbol{r}_{b}\right)
$$

with $p(\boldsymbol{r})$ being a function which typically is large for small arguments and small for large arguments. In this work, we use $p(\boldsymbol{r})=[\exp (1 / n r)-1-1 / n r]^{n}$ with $n=5$; instead of taking the limit $n \rightarrow \infty$, we take $n=5$ for performing the numerical integration accurately. Using $w_{a}(\boldsymbol{r})$, a function of space variables, $f(\boldsymbol{r})$, is divided into atomic components, $f_{a}(\boldsymbol{r})$, as follows:

$$
f(\boldsymbol{r})=\sum_{a} f_{a}(\boldsymbol{r})
$$

where $f_{a}(\boldsymbol{r})=w_{a}(\boldsymbol{r}) f(\boldsymbol{r})$. The integral of $f(\boldsymbol{r})$ over the whole solid, $I$, is also divided into atomic components, $I_{a}$, as follows:

$$
I=\sum_{a} I_{a}
$$

where

$$
I=\int f(\boldsymbol{r}) \mathrm{d} \boldsymbol{r}
$$

and

$$
I_{a}=\int f_{a}(\boldsymbol{r}) \mathrm{d} \boldsymbol{r} .
$$

Thus, using the spin magnetization associated with the ath atom, $\boldsymbol{m}_{a}(\boldsymbol{r})=w_{a}(\boldsymbol{r}) \boldsymbol{m}(\boldsymbol{r})$, we calculate the atomic spin magnetic moment, $\boldsymbol{M}_{a}^{\text {spin }}$ :

$$
\boldsymbol{M}_{a}^{\text {spin }}=\int \boldsymbol{m}_{a}(\boldsymbol{r}) \mathrm{d} \boldsymbol{r} .
$$

Also, using the Dirac current associated with the ath atom, $\boldsymbol{j}_{a}(\boldsymbol{r})=w_{a}(\boldsymbol{r}) \boldsymbol{j}(\boldsymbol{r})$, we calculate the atomic total magnetic moment, $\boldsymbol{M}_{a}^{\text {tot: }}$

$$
\boldsymbol{M}_{a}^{\mathrm{tot}}=\frac{1}{2 c} \int \boldsymbol{r} \times \boldsymbol{j}_{a}(\boldsymbol{r}) \mathrm{d} \boldsymbol{r} .
$$


Here, it may be worth mentioning again that $\boldsymbol{j}(\boldsymbol{r})$ consists of not only the orbital contribution but also the spin contribution. Accordingly, $\boldsymbol{M}_{a}^{\text {tot }}$ consists of both the orbital and spin contributions. Finally, we calculate the atomic orbital magnetic moment, $\boldsymbol{M}_{a}^{\text {orb}}$ :

$$
\boldsymbol{M}_{a}^{\mathrm{orb}}=\boldsymbol{M}_{a}^{\mathrm{tot}}-\boldsymbol{M}_{a}^{\mathrm{spin}} .
$$

An important point to be noted is that $\boldsymbol{M}_{a}^{\text {tot }}$ calculated with eq. (12) is independent of the choice of the origin of the position vector only if

$$
\int \boldsymbol{j}_{a}(\boldsymbol{r}) \mathrm{d} \boldsymbol{r}=\mathbf{0}
$$

We have checked that this condition is always satisfied for the calculated results given in the next section.

We here remark that the conventional method used in previous theoretical studies for calculating $M^{\text {orb }}$ is different from that used in this work although the method for calculating $M^{\text {spin }}$ is the same. The formula used previously for calculating $M^{\text {orb }}$ is the following: ${ }^{13)}$

$$
\boldsymbol{M}_{a}^{\text {orb }}=-e \operatorname{Re}\left(\sum_{p \in a} \sum_{q} \sum_{\nu} f_{\nu} C_{p \nu}^{*} C_{q \nu} \int \chi_{p}(\boldsymbol{r})^{\dagger} \beta \boldsymbol{l} \chi_{q}(\boldsymbol{r}) \mathrm{d} \boldsymbol{r}\right) .
$$

Here $\chi_{p}(\boldsymbol{r})$ are the basis functions employed in the calculations and $C_{p \nu}$ are the coefficients in the expansion of $\psi_{\nu}(\boldsymbol{r})$ with $\chi_{p}(\boldsymbol{r})$. Also, $\boldsymbol{l}$ represent the angular momentum operator, $\boldsymbol{r} \times \boldsymbol{p}$. It is important to note that eq. (15) is applicable only if we can evaluate $\boldsymbol{l} \chi_{q}(\boldsymbol{r})$ definitely; for example, it is impossible to calculate $M^{\text {orb }}$ with eq. (15) if the basis set includes plane waves. On the contrary, we can use eqs. (11)-(13) for calculating $M^{\text {orb }}$ with any type of basis function if the quality of the basis set is good.

$\mathrm{UX}$ crystallize in the $\mathrm{NaCl}$ structure exhibiting a strong magnetic anisotropy with an easy axis in the [111] direction. ${ }^{1)}$ The experimental lattice constants of US, USe, and UTe are 5.489, 5.740, and $6.155 \AA$, respectively. These experimental lattice constants were used in our calculations. We assumed that the magnetization axis is in the [111] direction, which was taken as the $z$ axis in our calculations. The basis functions adopted in the FFLCAO method consist of the following four-component atomic orbitals: $1 s, 2 s, 2 p, 3 s, 3 p, 3 d, 4 s, 4 p, 4 d, 4 f$, $5 s, 5 p, 5 d, 5 f, 6 s, 6 p, 6 d$, and $7 s$ orbitals of the neutral $\mathrm{U}$ atom, $5 f, 7 s$, and $7 p$ orbitals of the $\mathrm{U}^{2+}$ atom, $1 s, 2 s, 2 p, 3 s$, and $3 p$ atomic orbitals of the neutral $\mathrm{S}$ atom, and $3 s, 3 p$, and $3 d$ orbitals of the $\mathrm{S}^{2+}$ atom, $1 s, 2 s, 2 p, 3 s, 3 p, 3 d, 4 s$, and $4 p$ atomic orbitals of the neutral Se atom, and $4 s, 4 p$, and $4 d$ orbitals of the $\mathrm{Se}^{2+}$ atom, $1 s, 2 s, 2 p, 3 s, 3 p, 3 d, 4 s, 4 p, 4 d, 5 s$, and $5 p$ atomic orbitals of the neutral Te atom, and $5 s, 5 p$, and $5 d$ orbitals of the $\mathrm{Te}^{2+}$ atom. Also, the basis functions adopted in the FFMB method consist of the four-component atomic orbitals of neutral $\mathrm{U}$ and $X$ atoms used in the FFLCAO method and four-component plane waves, which are positive-energy solutions of the Dirac equation for a free electron. In this work, we chose the cut-off energy of the four-component plane waves to be $50 \mathrm{eV}$. This cut-off energy corresponds to about 40,50, and 60 four-component plane waves per each $\boldsymbol{k}$ point 
for US, USe, and UTe, respectively. In the FFLCAO method, it is necessary to use not only the atomic orbitals of neutral atoms but also those of positively charged atoms for describing the contraction of atomic orbitals associated with cohesion. In the FFMB method, the fourcomponent plane waves play the same role. We carried out real-space integration using 4644 points for the U atom, 2580 points for the S atom, 3096 points for the Se atom, and 3612 points for the Te atom. Also, we used the LSDA exchange-correlation potential represented by the Perdew-Zunger parameterization of Ceperly-Alder results. ${ }^{28,29)}$ The Brillouin-zone integration was carried out using the good-lattice-point method with $185 \boldsymbol{k}$ points. ${ }^{30)}$ These conditions of calculations were confirmed previously to be sufficient for studying the electronic properties including the magnetic ones. ${ }^{11,31)}$

\section{Results and discussion}

In Table I, we show the results of this work as well as those of previous theoretical and experimental studies. Listed in the table are the orbital, spin, and total magnetic moments of the $\mathrm{U}$ atom, $M_{\mathrm{U}}^{\text {orb }}, M_{\mathrm{U}}^{\text {spin }}$, and $M_{\mathrm{U}}^{\text {tot }}$, those of the $X$ atom, $M_{X}^{\text {orb }}, M_{X}^{\text {spin }}$, and $M_{X}^{\text {tot }}$, and their sums, $M_{\mathrm{U}+X}^{\mathrm{orb}}=M_{\mathrm{U}}^{\mathrm{orb}}+M_{X}^{\mathrm{orb}}, M_{\mathrm{U}+X}^{\mathrm{spin}}=M_{\mathrm{U}}^{\mathrm{spin}}+M_{X}^{\mathrm{spin}}$, and $M_{\mathrm{U}+X}^{\text {tot }}=M_{\mathrm{U}}^{\text {tot }}+M_{X}^{\text {tot }}$. In the first and second rows for each material, we show the magnetic moments obtained in this work using the FFLCAO and FFMB calculations with eqs. (11)-(13), respectively. It should be noted that the formula used for calculating $M^{\text {orb }}$ in all the previous theoretical studies is eq. (15).

We begin with the comparison between the magnetic moments obtained using the FFLCAO calculations and those obtained using the FFMB calculations, both employing eqs. (11)-(13). For example, the details of the results for US are as follows. The $M_{\mathrm{U}}^{\mathrm{orb}}, M_{\mathrm{S}}^{\mathrm{orb}}$, and $M_{\mathrm{U}+\mathrm{S}}^{\mathrm{orb}}$ obtained using the FFLCAO (FFMB) calculations are 2.59 (2.62), 0.18 (0.21), and $2.77(2.83) \mu_{\mathrm{B}}$, respectively. The $M_{\mathrm{U}}^{\mathrm{spin}}, M_{\mathrm{S}}^{\mathrm{spin}}$, and $M_{\mathrm{U}+\mathrm{S}}^{\text {spin }}$ obtained using the FFLCAO (FFMB) calculations are $-1.69(-1.72),-0.13(-0.12)$, and $-1.82(-1.84) \mu_{\mathrm{B}}$, respectively. The $M_{\mathrm{U}}^{\text {tot }}, M_{\mathrm{S}}^{\text {tot }}$, and $M_{\mathrm{U}+\mathrm{S}}^{\text {tot }}$ obtained using the FFLCAO (FFMB) calculations are $0.90(0.90)$, $0.05(0.09)$, and $0.95(0.99) \mu_{\mathrm{B}}$, respectively. We thus find that the differences between the results of the FFLCAO calculations and those of the FFMB calculations, at most $0.06 \mu_{\mathrm{B}}$, are considerably small. For USe and UTe, the differences are found to be much smaller, i.e., at most $0.02 \mu_{\mathrm{B}}$. In particular, it should be noted that $M^{\text {orb }}$ obtained using the FFLCAO calculations and those obtained using the FFMB calculations agree very well with each other. This means that the method with the Dirac current enables us to calculate $M^{\text {orb }}$ even if the basis set includes basis functions with no definite angular momenta if the quality of the basis set is good.

We next proceed to the comparison between the results of this work and those of the previous theoretical studies in which eq. (15) is used for calculating $M^{\text {orb }}$. Here, it may be helpful to classify these previous studies. So far, there have been a few studies that reported 
not only the magnetic moments of the $\mathrm{U}$ atom but also those of the $X$ atom. To our knowledge, our previous FFLCAO study and the relativistic augmented-spherical-wave (ASW) study are the only two that reported the magnetic moments of the $X$ atom explicitly; ${ }^{5,11)}$ the reason may be that the magnetic moments of the $X$ atom obtained in the other previous studies are negligibly small. Most of the previous studies reported the magnetic moments only of the $\mathrm{U}$ atom. Furthermore, some of them reported only the U $5 f$ contributions; in Table I, we show the U $5 f$ contributions in parentheses. However, the differences between them are often found to be very large; some of these differences exceed $0.5 \mu_{\mathrm{B}}$. This may be due to the fact that the decomposition of the magnetic moments into the contributions of the constituent atomic orbitals depends on the employed basis set. It should be noted that the decomposition of the magnetic moments into the contributions of atomic orbitals affects not only $M^{\text {orb }}$ but also even $M^{\text {spin }}$. With the above classification in mind, the most definite comparison of the results of this work can be made with those of our previous FFLCAO study and with those of the ASW study. In particular, since the sums of the magnetic moments of the $\mathrm{U}$ and $X$ atoms, i.e., $M_{\mathrm{U}+X}^{\mathrm{orb}}, M_{\mathrm{U}+X}^{\mathrm{spin}}$, and $M_{\mathrm{U}+X}^{\mathrm{tot}}$, are expected to be almost independent of the atomic partitioning scheme employed, the comparison of these quantities should be most meaningful. For this reason, we mainly consider $M_{\mathrm{U}+X}^{\text {orb }}, M_{\mathrm{U}+X}^{\text {spin }}$, and $M_{\mathrm{U}+X}^{\text {tot }}$ below.

We now compare the results of the present FFLCAO calculations with those of our previous FFLCAO study. ${ }^{11)}$ The comparison is very interesting because all the conditions of calculations are exactly the same except that the formula used previously is eq. (15). This enables us to examine the difference between the results calculated using eqs. (11)-(13) and those calculated using eq. (15) without any ambiguity. In particular, it should be noted that the difference in $M_{\mathrm{U}+X}^{\text {tot }}$ is entirely originated in $M_{\mathrm{U}+X}^{\text {orb }}$ because $M_{\mathrm{U}+X}^{\text {spin }}$ is exactly the same for both cases. It is found that $M_{\mathrm{U}+X}^{\mathrm{orb}}$ calculated with eqs. (11)-(13) is $2.77,3.06$, and $3.55 \mu_{\mathrm{B}}$ for US, USe, and UTe, respectively. On the other hand, $M_{\mathrm{U}+X}^{\mathrm{orb}}$ calculated with eq. (15) is 2.40, 2.71, and $3.18 \mu_{\mathrm{B}}$ for US, USe, and UTe, respectively. It is also found that $M_{\mathrm{U}+X}^{\mathrm{tot}}$ calculated with eqs. (11)-(13) is $0.95,1.16,1.47 \mu_{\mathrm{B}}$ for US, USe, and UTe, respectively. On the other hand, $M_{\mathrm{U}+X}^{\text {tot }}$ calculated with eq. (15) is $0.58,0.81$, and $1.10 \mu_{\mathrm{B}}$ for US, USe, and UTe, respectively. For both $M_{\mathrm{U}+X}^{\mathrm{orb}}$ and $M_{\mathrm{U}+X}^{\text {tot }}$, the differences between the results of the present FFLCAO calculations and those of the previous FFLCAO study are $0.37,0.35$, and $0.37 \mu_{\mathrm{B}}$ for US, USe, and UTe, respectively. We thus arrive at the conclusion that the method with the Dirac current, i.e., eqs. (11)-(13), gives larger $M_{\mathrm{U}+X}^{\mathrm{orb}}$ than the conventional method, i.e., eq. (15), does by nearly $0.4 \mu_{\mathrm{B}}$. The differences are not only negligible in $M_{\mathrm{U}+X}^{\mathrm{orb}}$ but also even crucial in $M_{\mathrm{U}+X}^{\text {tot }}$ because the magnitude of $M_{\mathrm{U}+X}^{\text {tot }}$ is much smaller than that of $M_{\mathrm{U}+X}^{\text {orb }}$ because of the antiparallel coupling between $M_{\mathrm{U}+X}^{\mathrm{orb}}$ and $M_{\mathrm{U}+X}^{\text {spin }}$. That is, $0.4 \mu_{\mathrm{B}}$ amounts to about $30 \%$ of $M_{\mathrm{U}+X}^{\mathrm{tot}}$.

It is worth mentioning that $M_{\mathrm{U}+X}^{\mathrm{orb}}, M_{\mathrm{U}+X}^{\text {spin }}$, and $M_{\mathrm{U}+X}^{\text {tot }}$ obtained in our previous FFLCAO 
study are in reasonable agreement with those obtained in the ASW study despite that the methodology employed in the ASW study is considerably different from ours; the differences in $M_{\mathrm{U}+X}^{\text {orb }}, M_{\mathrm{U}+X}^{\text {spin }}$, and $M_{\mathrm{U}+X}^{\text {tot }}$ are less than about $0.2 \mu_{\mathrm{B}}$ for all the materials. One point to be referred to may be that $M_{X}^{\text {tot }}$ obtained in the ASW study is opposite to those obtained using the present FFLCAO and FFMB calculations probably because the employed atomic partitioning scheme is different. Other than this discrepancy, the agreement between the results of our previous FFLCAO study and those of the ASW study is reasonable. We thus arrive at the same conclusion that $M_{\mathrm{U}+X}^{\text {tot }}$ obtained in the ASW study are smaller than those obtained in this work using the method with the Dirac current; the differences are again not negligible, i.e., about $0.2,0.3$, and $0.5 \mu_{\mathrm{B}}$ for US, USe, and UTe, respectively, as found from Table I.

Two of the previous theoretical studies reported the magnetic moments only of the $U$ atom: the full-potential linear muffin-tin-orbital (FPLMTO) study and the fully relativistic linear muffin-tin-orbital (RLMTO) study. ${ }^{6,7)}$ Here, although there remains an ambiguity, let us suppose that the magnetic moments of the $X$ atoms are negligible in their results. It is then found that, considering the differences in the employed methodologies, $M_{\mathrm{U}}^{\text {orb }}, M_{\mathrm{U}}^{\text {spin }}$, and $M_{\mathrm{U}}^{\text {tot }}$ obtained in both the FPLMTO and RLMTO studies are in acceptable agreement with $M_{\mathrm{U}+X}^{\text {orb }}$, $M_{\mathrm{U}+X}^{\text {spin }}$, and $M_{\mathrm{U}+X}^{\text {tot }}$ obtained in our previous FFLCAO study and also with those obtained in the ASW study; this indicates again that $M_{\mathrm{U}+X}^{\text {orb }}$ and $M_{\mathrm{U}+X}^{\text {tot }}$ calculated with eq. (15) are smaller by nearly $0.4 \mu_{\mathrm{B}}$ than those calculated using the method with the Dirac current.

Here, we also refer to the $\mathrm{U} 5 f$ contributions reported previously. It seems that the $\mathrm{U}$ $5 f$ contributions to $M_{\mathrm{U}}^{\mathrm{orb}}$ are slightly larger than $M_{\mathrm{U}}^{\mathrm{orb}}$ obtained in refs. $5-7,11$; the ASW study has shown that the $\mathrm{U} 5 f$ contribution to $M_{\mathrm{U}}^{\text {orb }}$ is larger by $0.1 \mu_{\mathrm{B}}$ than $M_{\mathrm{U}}^{\text {orb }}$ itself. On the other hand, it seems that the $\mathrm{U} 5 f$ contributions to $M_{\mathrm{U}}^{\mathrm{spin}}$ are almost the same or slightly smaller than $M_{\mathrm{U}}^{\text {spin }}$ obtained in refs. 5-7,11; the ASW study has shown that the U $5 f$ contribution to $M_{\mathrm{U}}^{\text {spin }}$ is smaller by $0.1 \mu_{\mathrm{B}}$ than $M_{\mathrm{U}}^{\text {spin }}$ itself. As a result, it seems that the $\mathrm{U} 5 f$ contributions to $M_{\mathrm{U}}^{\text {tot }}$ are larger by $0.2-0.3 \mu_{\mathrm{B}}$ than $M_{\mathrm{U}}^{\text {tot }}$ obtained in refs. $5-7,11$, as indicated by the results of the ASW study. Thus, we believe that, considering the differences in the employed methodologies, there are no serious contradictions among the results of the previous theoretical studies, which employed eq. (15) for calculating $M^{\text {orb }}$.

We now consider the calculated magnetic moments of the $X$ atom. As shown in Table I, our present FFLCAO and FFMB calculations give not only $M_{X}^{\text {spin }}$ but also non-negligible $M_{X}^{\text {orb }}$. It seems interesting to note that $M_{X}^{\text {orb }}$ is parallel to $M_{\mathrm{U}}^{\text {orb }}$ and the $M_{X}^{\text {spin }}$ is also parallel to $M_{\mathrm{U}}^{\mathrm{spin}}$. The results indicate that induced on the $X$ atom by the surrounding $\mathrm{U}$ atoms is not only the spin polarization but also the circulation of the orbital part of the Dirac current. This phenomenon was not observed in our previous FFLCAO study, where we found vanishingly small $M_{X}^{\text {orb }}$ and $M_{X}^{\text {spin }}$. It should also be pointed out that, in contrast to the results of this 
work, the ASW study reported that $M_{X}^{\text {orb }}$ are antiparallel to $M_{\mathrm{U}}^{\text {orb }}$. These discrepancies may also be attributed to the differences in the atomic partitioning scheme employed.

Finally, we compare $M^{\text {tot }}$ obtained in this work with the experimental ones. So far, $M^{\text {tot }}$ of $\mathrm{UX}$ have been measured using two experimental methods. One is the measurement of the bulk magnetic moment at saturation; this method directly measures $M^{\text {tot }}$ of a single crystal, which may correspond to $M_{\mathrm{U}+X}^{\mathrm{tot}}$. The other is the neutron diffraction measurement; in contrast to the former method, this method estimates the U $5 f$ contribution. The bulk magnetic moment at saturation is always smaller than the magnetic moment obtained using the neutron diffraction; for US, USe, and UTe, the former results are 1.55, 1.81, and $1.91 \mu_{\mathrm{B}}$, respectively, while the latter results are $1.70,2.0$, and $2.25 \mu_{\mathrm{B}}$, respectively. We compare $M_{\mathrm{U}+X}^{\text {tot }}$ obtained in this work with the bulk magnetic moment at saturation because this quantity is less ambiguous than the $\mathrm{U} 5 f$ contribution. We find that the results of this work are smaller by about 0.6 $\mu_{\mathrm{B}}$ for all the materials. We conclude that, even if eqs. (11)-(13) are used instead of eq. (15), the discrepancy is still large although considerably reduced. One of the possible origins of the discrepancy is that the measurement of the bulk magnetic moment involves the contribution of the surface current which may be different from the one expected from the calculated bulk properties. Another possible origin is the deficiency of the LSDA as claimed in the previous theoretical studies. Furthermore, there is a possibility that the measured magnetic moments cannot be reproduced as far as we restrict ourselves within SDFT; if this is the case, it may be CDFT that we need beyond SDFT. To resolve the disagreement between the calculated and measured magnetic moments, further theoretical studies are needed in the future.

\section{Conclusions}

We have studied $M^{\text {orb }}, M^{\text {spin }}$, and $M^{\text {tot }}$ in UX using the FFLCAO and FFMB methods. In particular, $M^{\text {orb }}$ are calculated with the Dirac current. We have shown that $M^{\text {orb }}$ calculated using the FFLCAO method and those calculated using the FFMB method agree very well with each other. This means that, in contrast to the conventional method, the method with the Dirac current enables us to calculate $M^{\text {orb }}$ even if the basis set includes basis functions with no definite angular momenta, e.g., the plane waves in the FFMB method. Furthermore, we have found that $M^{\text {orb }}$ obtained in this work are larger by nearly $0.4 \mu_{\mathrm{B}}$ than those obtained using the conventional method. This is crucial because the resultant differences in $M^{\text {tot }}$ are about $30 \%$. 
Table I. Orbital, spin, and total magnetic moments (in unit of $\mu_{\mathrm{B}}$ ) of $\mathrm{U}$ and $X$ atoms in $\mathrm{U} X$.

\begin{tabular}{|c|c|c|c|c|c|c|c|c|c|c|}
\hline & & \multicolumn{3}{|c|}{$M^{\text {orb }}$} & \multicolumn{3}{|c|}{$M^{\text {spin }}$} & \multicolumn{3}{|c|}{$M^{\text {tot }}$} \\
\hline & & $\mathrm{U}$ & $X$ & $\mathrm{U}+X$ & $\mathrm{U}$ & $X$ & $\mathrm{U}+X$ & $\mathrm{U}$ & $X$ & $\mathrm{U}+X$ \\
\hline \multirow[t]{12}{*}{ US } & FFLCAO $^{a)}$ & 2.59 & 0.18 & 2.77 & -1.69 & -0.13 & -1.82 & 0.90 & 0.05 & 0.95 \\
\hline & $\mathrm{FFMB}^{\mathrm{b}}$ & 2.62 & 0.21 & 2.83 & -1.72 & -0.12 & -1.84 & 0.90 & 0.09 & 0.99 \\
\hline & FFLCAO $^{\mathrm{c})}$ & 2.40 & 0.00 & 2.40 & -1.83 & 0.01 & -1.82 & 0.57 & 0.01 & 0.58 \\
\hline & $\mathrm{ASW}^{\mathrm{d})}$ & 2.5 & -0.07 & 2.4 & -1.6 & -0.06 & -1.7 & 0.9 & -0.13 & 0.8 \\
\hline & FPLMTO $^{\mathrm{e}}$ & 2.39 & & & -1.87 & & & 0.52 & & \\
\hline & RLMTO $^{\mathrm{f}}$ & 2.14 & & & -1.53 & & & 0.60 & & \\
\hline & $\mathrm{ASW}^{\mathrm{d})}$ & $(2.6)$ & & & $(-1.5)$ & & & (1.1) & & \\
\hline & LMTOg) & $(3.2)$ & & & $(-2.1)$ & & & (1.1) & & \\
\hline & RLAPWh) $^{\mathrm{h}}$ & $(2.58)$ & & & $(-1.70)$ & & & $(0.88)$ & & \\
\hline & FLAPW $^{\mathrm{i})}$ & $(2.33)$ & & & $(-1.60)$ & & & $(0.73)$ & & \\
\hline & Expt.j) & & & & & & & & & 1.55 \\
\hline & Expt. ${ }^{\mathrm{k})}$ & & & & & & & & & $(1.70)$ \\
\hline \multirow[t]{12}{*}{ USe } & FFLCAO $^{a)}$ & 2.89 & 0.17 & 3.06 & -1.79 & -0.11 & -1.90 & 1.10 & 0.06 & 1.16 \\
\hline & $\mathrm{FFMB}^{\mathrm{b}}$ & 2.88 & 0.18 & 3.06 & -1.79 & -0.10 & -1.89 & 1.09 & 0.08 & 1.17 \\
\hline & FFLCAO $^{\mathrm{c}}$ & 2.71 & 0.00 & 2.71 & -1.92 & 0.02 & -1.90 & 0.79 & 0.02 & 0.81 \\
\hline & $\mathrm{ASW}^{\mathrm{d})}$ & 2.8 & -0.07 & 2.7 & -1.8 & -0.06 & -1.9 & 1.0 & -0.13 & 0.9 \\
\hline & FPLMTO $^{\mathrm{e}}$ & 2.68 & & & -2.01 & & & 0.67 & & \\
\hline & RLMTO $^{\mathrm{f})}$ & 2.54 & & & -1.75 & & & 0.79 & & \\
\hline & $\mathrm{ASW}^{\mathrm{d})}$ & $(2.9)$ & & & $(-1.7)$ & & & $(1.2)$ & & \\
\hline & $\mathrm{LMTO}^{\mathrm{g})}$ & $(3.4)$ & & & $(-2.4)$ & & & (1.0) & & \\
\hline & RLAPW $^{\mathrm{h})}$ & $(3.11)$ & & & $(-1.96)$ & & & $(1.15)$ & & \\
\hline & FLAPW $^{\mathrm{i})}$ & $(2.92)$ & & & $(-1.94)$ & & & $(0.98)$ & & \\
\hline & Expt.j) & & & & & & & & & 1.81 \\
\hline & Expt. ${ }^{1)}$ & & & & & & & & & $(2.0)$ \\
\hline
\end{tabular}


Table I. (Continued)

\begin{tabular}{|c|c|c|c|c|c|c|c|c|c|c|}
\hline & & \multicolumn{3}{|c|}{$M^{\text {orb }}$} & \multicolumn{3}{|c|}{$M^{\text {spin }}$} & \multicolumn{3}{|c|}{$M^{\text {tot }}$} \\
\hline & & $\mathrm{U}$ & $X$ & $\mathrm{U}+X$ & $\mathrm{U}$ & $X$ & $\mathrm{U}+X$ & $\mathrm{U}$ & $X$ & $\mathrm{U}+X$ \\
\hline \multirow[t]{12}{*}{$\mathrm{UTe}$} & FFLCAO $^{a)}$ & 3.40 & 0.15 & 3.55 & -2.00 & -0.08 & -2.08 & 1.40 & 0.07 & 1.47 \\
\hline & FFMB $^{\mathrm{b})}$ & 3.39 & 0.16 & 3.55 & -2.01 & -0.09 & -2.10 & 1.38 & 0.07 & 1.45 \\
\hline & FFLCAO $^{\mathrm{c})}$ & 3.18 & 0.00 & 3.18 & -2.11 & 0.03 & -2.08 & 1.07 & 0.03 & 1.10 \\
\hline & $\mathrm{ASW}^{\mathrm{d})}$ & 3.4 & -0.08 & 3.3 & -2.2 & -0.08 & -2.3 & 1.2 & -0.16 & 1.0 \\
\hline & FPLMTO $^{\mathrm{e}}$ & 3.23 & & & -2.35 & & & 0.88 & & \\
\hline & RLMTO $\left.^{\mathrm{f}}\right)$ & 3.12 & & & -2.12 & & & 1.00 & & \\
\hline & $\mathrm{ASW}^{\mathrm{d})}$ & $(3.5)$ & & & $(-2.0)$ & & & $(1.5)$ & & \\
\hline & $\mathrm{LMTO}^{\mathrm{g})}$ & $(3.4)$ & & & $(-2.6)$ & & & $(0.8)$ & & \\
\hline & RLAPW $\left.{ }^{\mathrm{h}}\right)$ & $(3.71)$ & & & $(-2.31)$ & & & $(1.40)$ & & \\
\hline & FLAPW $\left.{ }^{i}\right)$ & $(3.42)$ & & & $(-2.22)$ & & & $(1.20)$ & & \\
\hline & Expt.j) & & & & & & & & & 1.91 \\
\hline & Expt. ${ }^{1)}$ & & & & & & & & & $(2.25)$ \\
\hline
\end{tabular}

a) This work; fully relativistic full-potential LCAO calculations.

b) This work; fully relativistic full-potential mixed-basis calculations.

c) Ref. 11; fully relativistic full-potential LCAO calculations.

d) Ref. 5; relativistic augmented-spherical-wave calculations using a second variational method.

e) Ref. 6; full-potential linear muffin-tin-orbital calculations using a second variational method.

f) Ref. 7; fully relativistic linear muffin-tin-orbital calculations.

g) Ref. 8; relativistic linear muffin-tin-orbital calculations using a second variational method.

h) Ref. 9; fully relativistic linearized-augmented-plane-wave calculations.

i) Ref. 10; full-potential linearized-augmented-plane-wave calculations using a second variational method.

j) Ref. 2; bulk magnetic moment at saturation.

k) Ref. 3; neutron diffraction.

l) Ref. 4; neutron diffraction. 


\section{References}

1) Handbook on the Physics and Chemistry of the Actinides, ed. A. J. Freeman and G. H. Lander (North-Holland, Amsterdam, 1985).

2) J. M. Fournier and R. Tróc: in Handbook on the Physics and Chemistry of the Actinides, ed. A. J. Freeman and G. H. Lander (North-Holland, Amsterdam, 1985) Vol. 2, p. 239.

3) F. A. Wedgwood: J. Phys. C: Solid State Phys. 5 (1972) 2427.

4) F. A. Wedgwood and M. Kuznietz: J. Phys. C: Solid State Phys. 5 (1972) 3012.

5) T. Kraft, P. M. Oppeneer, V. N. Antonov, and H. Eschrig: Phys. Rev. B 52 (1995) 3561.

6) E. M. Collins, N. Kioussis, S. P. Lim, and B. R. Cooper: J. Appl. Phys. 85 (1999) 6226.

7) V. N. Antonov, B. N. Harmon, O. V. Andryushchenko, L. V. Bekenev, and A. N. Yaresko: Low Temp. Phys. 30 (2004) 305.

8) M. S. S. Brooks: Phisyca B 130 (1985) 6.

9) H. Yamagami: J. Phys. Soc. Jpn. 67 (1998) 3176.

10) T. Shishidou and T. Oguchi: Phys. Rev. B 62 (2000) 11747.

11) S. Suzuki, T. Ariizumi, and M.-F. Li: J. Phys. Soc. Jpn. 78 (2009) 074715.

12) M. S. S. Brooks and P. J. Kelly: Phys. Rev. Lett. 51 (1983) 1708.

13) M. Singh, J. Callaway, and C. S. Wang: Phys. Rev. B 14 (1976) 1214.

14) J. D. Jackson: Classical Electrodynamics (Wiley, New York, 1975).

15) A. D. Becke: J. Chem. Phys. 88 (1988) 2547.

16) B. Delley: J. Chem. Phys. 92 (1990) 508.

17) S. Suzuki and K. Nakao: J. Phys. Soc. Jpn. 66 (1997) 3881.

18) G. Vignale and M. Rasolt: Phys. Rev. Lett. 59 (1987) 2360.

19) G. Vignale and M. Rasolt: Phys. Rev. B 37 (1988) 10685.

20) K. Capelle and E. K. U. Gross: Phys. Rev. Lett. 78 (1997) 1872.

21) M. Higuchi and A. Hasegawa: J. Phys. Soc. Jpn. 66 (1997) 149.

22) M. Higuchi and A. Hasegawa: J. Phys. Soc. Jpn. 67 (1998) 2037.

23) K. Higuchi and M. Higuchi: Phys. Rev. B 74 (2006) 195122.

24) H. Ebert, M. Battocletti, and E. K. U. Gross: Europhys. Lett. 40 (1997) 545.

25) S. Suzuki and K. Nakao: J. Phys. Soc. Jpn. 68 (1999) 1982.

26) W. Greiner: Relativistic Quantum Mechanics (Springer-Verlag, Berlin, 1990).

27) W. Gordon: Z. Phys. 50 (1928) 630.

$28)$ D. M. Ceperley and B. J. Alder: Phys. Rev. Lett. 45566 (1980) 566.

29) J. P. Perdew and A. Zunger: Phys. Rev. B 23 (1981) 5048.

30) L.-K. Hua and Y. Wang: Applications of Number Theory to Numerical Analysis (Springer-Verlag, Berlin, 1981).

31) S. Suzuki, M.-F. Li, and T. Ariizumi: J. Phys. Soc. Jpn. 77 (2008) 074703. 